\title{
Expression of claudin-4 (CLDN4) mRNA in intraductal papillary mucinous neoplasms of the pancreas
}

\author{
Kosuke Tsutsumi, Norihiro Sato, Lin Cui, Kazuhiro Mizumoto, Yoshihiko Sadakari, \\ Hayato Fujita, Kenoki Ohuchida, Takao Ohtsuka, Shunichi Takahata and Masao Tanaka \\ Department of Surgery and Oncology, Graduate School of Medical Sciences, Kyushu University, \\ Fukuoka, Japan
}

\begin{abstract}
Claudin-4, encoding a protein for tight junction formation and function, is highly overexpressed in pancreatic ductal adenocarcinoma and is also associated with invasive adenocarcinomas arising in intraductal papillary mucinous neoplasms of the pancreas. However, the expression pattern of claudin-4 during neoplastic progression of intraductal papillary mucinous neoplasms remains unknown. Using quantitative real-time reverse transcriptionPCR, we analyzed claudin-4 mRNA in a panel of 14 pancreatic cancer cell lines and in formalin-fixed paraffinembedded tissues from $\mathbf{8 0}$ patients with intraductal papillary mucinous neoplasms of different histological grades and papillary subtypes. Increased expression of claudin- 4 was confirmed in all the pancreatic cancer cell lines tested as compared with normal ductal epithelial cells and fibroblast cultures. The claudin-4 expression was significantly higher in high-grade intraductal papillary mucinous neoplasms (borderline neoplasm and carcinoma) than in low-grade intraductal papillary mucinous neoplasms (adenoma) $(P<0.0001)$. In addition, claudin-4 mRNA levels were significantly higher in intestinal-type intraductal papillary mucinous neoplasms than in non-intestinaltype intraductal papillary mucinous neoplasms based on papillary subclassification $(P<0.0001)$. Our findings suggest that claudin-4 expression is associated with neoplastic progression of intraductal papillary mucinous neoplasms and, especially, with a distinct pathway to intestinal differentiation.
\end{abstract}

Modern Pathology (2011) 24, 533-541; doi:10.1038/modpathol.2010.218; published online 19 November 2010

Keywords: claudin-4 (CLDN4); formalin-fixed paraffin-embedded tissues; high-grade intraductal papillary mucinous neoplasms; intestinal-type intraductal papillary mucinous neoplasms; intraductal papillary mucinous neoplasms (IPMNs); pancreatic juices

Intraductal papillary mucinous neoplasms are multimodal entities with a wide spectrum of histopathological dysplasia. According to the criteria established by the World Health Organization (WHO), intraductal papillary mucinous neoplasms are classified into, adenoma defined as intraductal papillary mucinous neoplasm with slight or no dysplasia, borderline neoplasm as moderate dysplasia, carcinoma in situ (CIS) as severe dysplasia without invasion, and invasive carcinoma derived from intraductal papillary mucinous neoplasm. ${ }^{1}$ Intraductal papillary mucinous neoplasms are considered to progress sequentially from adenoma

Correspondence: Dr N Sato, MD, PhD, Department of Surgery and Oncology, Graduate School of Medical Sciences, Kyushu University, 3-1-1 Maidashi, Fukuoka 812-8582, Japan.

E-mail: norisato@fukuoka-med.jrc.or.jp

Received 18 July 2010; revised 14 October 2010; accepted 14

October 2010; published online 19 November 2010 to carcinoma in a stepwise fashion. ${ }^{2}$ Compared with non-invasive intraductal papillary mucinous neoplasms, invasive carcinomas derived from intraductal papillary mucinous neoplasms have a poor prognosis. ${ }^{3}$ Intraductal papillary mucinous neoplasms are also classified into several subtypes based on morphological features and immunohistochemical findings of mucin expression regardless of the histological grade, including gastric type, intestinal type, pancreatobiliary type, and oncocytic type. ${ }^{4,5}$ In general, most adenomas consist of gastrictype intraductal papillary mucinous neoplasms. However, intestinal-type intraductal papillary mucinous neoplasms tend to be found in high-grade intraductal papillary mucinous neoplasms of borderline neoplasm and carcinoma, notably colloid carcinoma. $^{2}$

According to the International Consensus Guidelines, ${ }^{6}$ all main duct intraductal papillary mucinous neoplasms, including mixed type, and branch duct 
intraductal papillary mucinous neoplasms suspected of malignancy are candidates for surgery. Although some clinical and imaging features, including dilatation of the main pancreatic duct, the presence of intramural nodules, cyst size of more than $30 \mathrm{~mm}$, and the presence of symptoms, have been shown to predict malignancy in intraductal papillary mucinous neoplasms, it is sometimes difficult to accurately diagnose the grade of malignancy of intraductal papillary mucinous neoplasms preoperatively by these predictors. Therefore, efforts are underway to identify novel markers, such as molecular biological markers, to preoperatively predict the grade and malignant potential of intraductal papillary mucinous neoplasms.

Genetic alterations in intraductal papillary mucinous neoplasms have been reported to involve $K$-ras mutation, ${ }^{7,8}$ and inactivation of several tumor-suppressor genes such as p53,p16, DPC4/Smad $4^{7,9,10}$ and $S T K 11 / L K B 1 .{ }^{11}$ In addition to these genetic events, aberrant $\mathrm{CpG}$ island hypermethylation is a frequent event in intraductal papillary mucinous neoplasms. ${ }^{12,13}$ More recently, a large-scale gene expression profiling using oligonucleotide microarrays identified a cluster of genes that were significantly overexpressed in intraductal papillary mucinous neoplasms. ${ }^{14}$ Among the genes identified, claudin-4 $(C L D N 4), C X C R 4, S 100 A 4$, and mesothelin were highly expressed in intraductal papillary mucinous neoplasms associated with an invasive carcinoma than in those without an invasive carcinoma. ${ }^{14}$

$C L D N 4$, encoding a transmembrane protein involved in tight junction formation and function, contributes to maintaining epithelial cell polarity and establishing the intercellular barrier. CLDN4 has been shown to be overexpressed in a variety of cancers, including ovarian carcinoma, ${ }^{15}$ breast carcinoma, ${ }^{16}$ prostate cancer, ${ }^{17}$ and pancreatic ductal adenocarcinoma. ${ }^{18-22}$ Importantly, CLDN4 has been reported as having an important role in the growth, invasion, and metastasis of pancreatic cancer. CLDN4 is also known to function as a high-affinity receptor for Clostridium perfringens enterotoxin $(\mathrm{CPE})^{23}$ and is, therefore, expected to be a therapeutic target. ${ }^{18} C L D N 4$ expression was also found in a subset of intraductal papillary mucinous neoplasms, but its expression pattern during neoplastic progression of intraductal papillary mucinous neoplasms remains unknown.

The aim of the present study was to elucidate the expression pattern of CLDN4 mRNA in intraductal papillary mucinous neoplasms with different histological grades and papillary subtypes.

\section{Materials and methods}

\section{Cultured Cells and Pancreatic Tissues as the Clinical Sample}

In all, 14 pancreatic cancer cell lines, AsPC-1, BxPC-3, KP-1N, KP-2, KP-3, PANC-1, SUIT-2 (provided by Dr H Iguchi, National Shikoku Cancer Center, Matsuyama, Japan), NOR-P1, ${ }^{24}$ CAPAN-1, CAPAN-2, CFPAC-1, H48N, HS766T and SW1990 (American Type Culture Collection, Manassas, VA, USA), 7 primary cultures of fibroblasts derived from 4 pancreatic ductal adenocarcinomas and 3 pancreatic normal tissues (established in our laboratory), and a human primary normal pancreatic epithelial cell line (Cell Systems, Kirkland, WA, USA) were used in the present study. Cells were maintained as described previously. ${ }^{25}$

We used quantitative real-time reverse transcription-PCR (qRT-PCR) to determine CLDN4 mRNA in formalin-fixed paraffin-embedded tissues of intraductal papillary mucinous neoplasms. In all, 80 formalin-fixed paraffin-embedded tissue samples of intraductal papillary mucinous neoplasms and 8 formalin-fixed paraffin-embedded tissue samples of pancreatic ductal adenocarcinomas obtained from patients undergoing pancreatic resection at our department (Kyushu University Hospital, Fukuoka, Japan) from 1987 to 2008 were selected for the present study based on the availability of sufficient quantities of neoplastic cells. These intraductal papillary mucinous neoplasms were categorized as adenoma, borderline neoplasm, carcinoma in situ (CIS), and invasive carcinoma by the WHO histological classification. ${ }^{1}$ For comparison, intraductal papillary mucinous neoplasms were simply divided into low-grade intraductal papillary mucinous neoplasms (adenoma) and high-grade intraductal papillary mucinous neoplasms (borderline neoplasm, CIS, and invasive carcinoma). Intraductal papillary mucinous neoplasms were also classified into four groups, including gastric, intestinal, pancreatobiliary and oncocytic types by the recently suggested subclassification system. ${ }^{4}$ Intraductal papillary mucinous neoplasms that could not be categorized into any of the four subtypes were defined as unclassified type. The gastric type, pancreatobiliary type, oncocytic type, and unclassified type were categorized as the non-intestinal-type intraductal papillary mucinous neoplasm and compared with the intestinal-type intraductal papillary mucinous neoplasm. Furthermore, six pancreatic juice samples obtained by endoscopic retrograde pancreatography for preoperative cytology were also analyzed.

Written informed consent was obtained from all patients, and the study was approved by the surveillance committee of our institution and conducted according to the Helsinki declaration.

\section{Macrodissection and RNA Extraction}

Formalin-fixed paraffin-embedded tissue samples were cut into $10-\mu \mathrm{m}$ thick sections and one of the sections was processed for 'temporary $\mathrm{H}$ and $\mathrm{E}$ stain' without the process of penetration and inclusion to identify the distribution of neoplastic and 
non-neoplastic cells. The number of slices required for RNA extraction varied among cases and was determined according to the estimated area of the neoplastic cells on $\mathrm{H}$ and $\mathrm{E}$ staining. Macrodissection was done using a small blade. By removing the non-neoplastic component as much as possible, only the neoplastic component was selectively isolated to obtain a high neoplastic cellularity. Total RNA was extracted from the isolated cells using the RNeasy Formalin-fixed paraffin-embedded Kit (Qiagen, Tokyo, Japan) according to kit instructions. In some cases, both epithelial and stromal tissue components were selectively isolated to investigate the difference of CLDN4 mRNA expression between these two components. The mirVana PARIS Kit (Ambion, Austin, USA) was used for the extraction of total RNA from pancreatic juice samples following the instruction manual.

\section{Quantitative Assessment of CLDN4 mRNA Level by Real-Time RT-PCR}

One-step quantitative real-time RT-PCR was performed using the QuantiTect SYBR Green RT-PCR kit (Qiagen) with a Chromo4 and the CFX384 RealTime PCR Detection System (Bio-Rad Laboratories, Hercules, CA, USA). Specifically, the reaction mixture was first incubated at $50{ }^{\circ} \mathrm{C}$ for $30 \mathrm{~min}$ to allow for reverse transcription. PCR was initiated with one cycle of $95^{\circ} \mathrm{C}$ for $15 \mathrm{~min}$ to activate the modified Taq polymerase followed by 40 cycles of $94{ }^{\circ} \mathrm{C}$ for $15 \mathrm{~s}, 55^{\circ} \mathrm{C}$ for $30 \mathrm{~s}, 72^{\circ} \mathrm{C}$ for $30 \mathrm{~s}$, and by one cycle of $60{ }^{\circ} \mathrm{C}$ for $5 \mathrm{~s},+0.1{ }^{\circ} \mathrm{C} / \mathrm{sec}$ to $95{ }^{\circ} \mathrm{C}$ for melting analysis. In addition, we designed specific primers as follows: CLDN4, 5'-GAGGGCCTATGGATGAACTG$3^{\prime}$ (forward) and 5'-AGCAGCGAGTCGTACACCTT-3' (reverse); $18 S$ rRNA, 5'-CTTTCGAGGCCCTGTAAT TG- $3^{\prime}$ (forward) and 5'-CCTCCAATGGATCCTCG TTA-3' (reverse). The quantitative range of threshold cycles observed was 20-35 cycles for CLDN4 primers and 10-30 cycles for $18 S$ rRNA primers. PCR was repeated at least twice in all samples, and three times if the sample showed more than $10 \%$ deviation in RT-PCR values. The level of CLDN4 mRNA expression was calculated from a standard curve generated with total RNA from the SUIT-2 pancreatic cancer cell line. Expressions of CLDN4 mRNA were normalized to that of $18 S$ rRNA. Results are presented as a box-andwhisker plot in formalin-fixed paraffin-embedded tissue samples, and as dots (individual levels) and a horizontal line (average level) in pancreatic juice samples.

\section{Statistical Analysis}

All statistical analyses were performed with JMP statistical software (version 6.0.3; SAS, Cary, NC, USA). As normal distributions were not obtained, data were analyzed by Wilcoxon rank-sum test. Statistical differences were considered significant when $P$-value was less than 0.05 .

\section{Results}

Quantitative Analysis of CLDN4 mRNA Levels in 14 Pancreatic Cancer Cell Lines, 7 Primary Cultures of Pancreatic Fibroblasts, and a Human Primary Normal Pancreatic Epithelial Cell Line

Previous studies have shown overexpression of CLDN4 mRNA in association with gene hypomethylation in pancreatic cancer cell lines. ${ }^{20,26}$ Using quantitative real time RT-PCR, we first determined the CLDN4 mRNA expression levels in a panel of 14 pancreatic cancer cell lines, a human primary normal pancreatic epithelial cell line, and 7 primary cultures of pancreatic fibroblasts. Increased expression of CLDN4 was found in all the pancreatic cancer cell lines tested as compared with the levels in a human primary normal pancreatic epithelial cell line and fibroblast cultures (Figure 1).

\section{Patient Characteristics and Clinicopathological Features of Intraductal Papillary Mucinous Neoplasms}

A total of 80 samples of intraductal papillary mucinous neoplasms were selected for macrodissection and CLDN4 mRNA expression analysis. These patients with intraductal papillary mucinous neoplasms consisted of 48 men and 32 women with a mean age of 67.5 years (range, 46-84 years). There were 21 main duct intraductal papillary mucinous neoplasms and 59 branch duct intraductal papillary mucinous neoplasms. They included 36 intraductal papillary mucinous adenomas, 19 intraductal papillary mucinous borderline neoplasms, 14 CISs, and 11 invasive carcinomas derived from intraductal papillary mucinous neoplasm. Papillary subclassification identified gastric type in 45 cases, intestinal type in 25 , pancreatobiliary type in 5 , oncocytic type in 1 , and unclassified type in the remaining 4.

\section{Quantitative Analysis of CLDN4 mRNA Expression in Intraductal Papillary Mucinous Neoplasms}

Macrodissection was successfully done to obtain a high neoplastic cellularity of $\sim 90 \%$ (Figure 2). We confirmed that non-neoplastic cells, including acinar, islet cells, and stromal fibroblasts showed lower levels of CLDN4 mRNA expression as compared with those of neoplastic cells by separately isolating the neoplastic and non-neoplastic components in the same samples (Supplementary Figure 1 and data not shown). Therefore, the effect of contamination of non-neoplastic cells on the expression level of CLDN4 in neoplastic cells can be negligible.

We were able to successfully PCR amplify all the 80 samples of intraductal papillary mucinous neoplasms. The expression level of CLDN4 mRNA was variable among the cases and seems higher along the progression of intraductal papillary mucinous neoplasms. When the relationship between the CLDN4 mRNA expression level and histological 


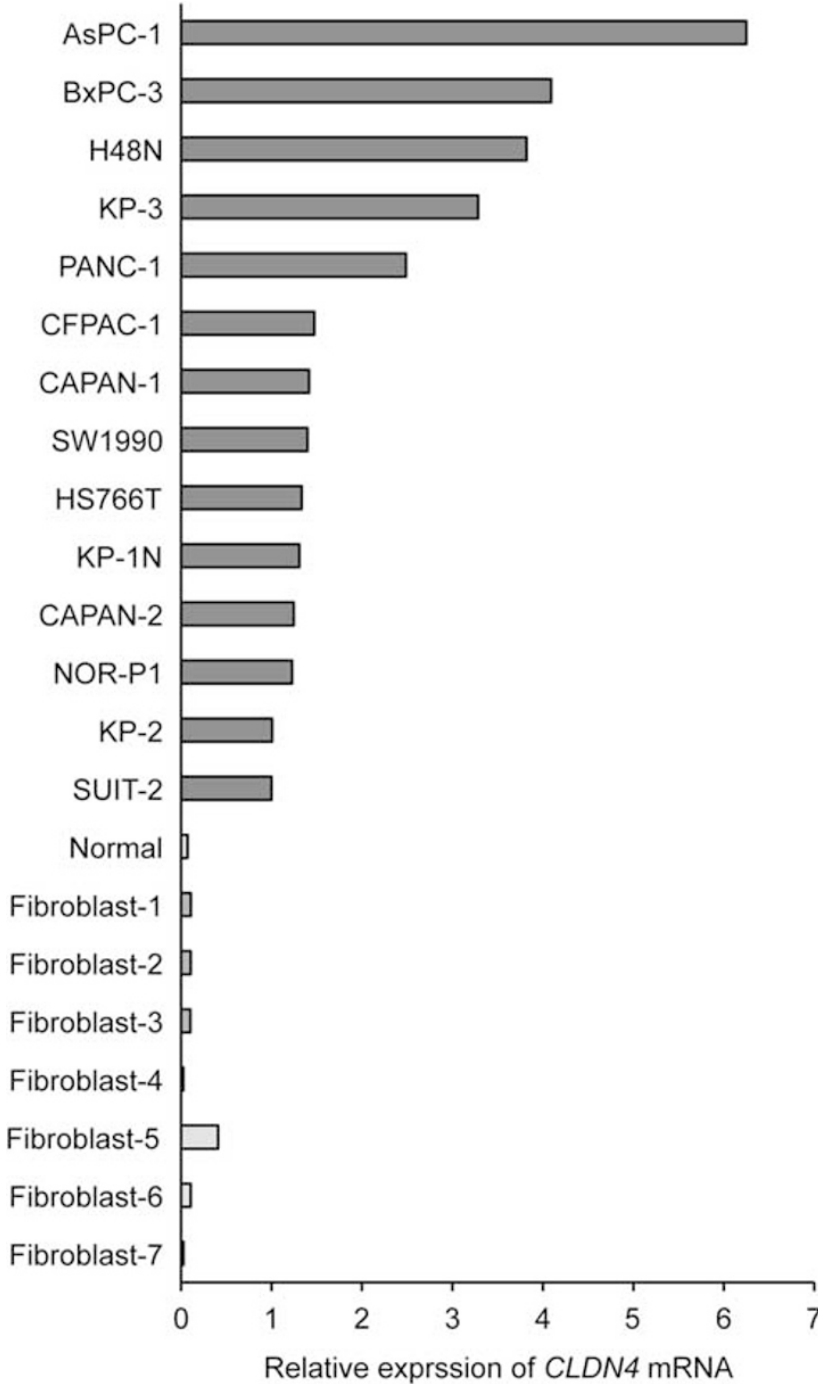

Figure $1 C L D N 4$ mRNA expression in 14 pancreatic cancer cell lines, a human primary normal pancreatic epithelial cell line (normal), and seven primary fibroblasts derived from pancreatic cancers (fibroblast 1-4) and pancreatic normal tissues (fibroblast 5-7). Increased expression of CLDN4 are found in all the pancreatic cancer cell lines tested as compared with the levels in a human primary normal pancreatic epithelial cell line and fibroblast cultures.

grade was analyzed, the expression levels of $C L D N 4$ mRNA in intraductal papillary mucinous borderline neoplasm, CIS, and invasive carcinoma derived from intraductal papillary mucinous neoplasm were significantly higher than that in intraductal papillary mucinous adenoma (Figure 3a, adenoma versus borderline neoplasm; $P=0.0055$, adenoma versus CIS; $P=0.0065$, adenoma versus invasive carcinoma; $P=0.0012$ ). When these intraductal papillary mucinous neoplasms were divided simply into high-grade intraductal papillary mucinous neoplasms (borderline neoplasm and higher grades, $n=44$ ) and low-grade intraductal papillary mucinous neoplasms (adenoma, $n=36$ ), $C L D N 4$ mRNA levels were significantly higher in high-grade intraductal papillary mucinous neoplasms than in low-grade intraductal papillary mucinous neoplasms (Figure 3b, $P<0.0001$ ).

We then examined whether there was any correlation between papillary subtype and CLDN4 expression in intraductal papillary mucinous neoplasms. After excluding one oncocytic-type and four unclassifiedtype intraductal papillary mucinous neoplasms, the $C L D N 4$ expression levels were compared among the three papillary subtypes (gastric, intestinal, and pancreatobiliary types). The CLDN4 expression levels were significantly higher in intestinal-type intraductal papillary mucinous neoplasms than in the others (Figure 3c, gastric type versus intestinal type; $P<0.0001$, intestinal type versus pancreatobiliary type; $P=0.0422$ ). There was no difference in $C L D N 4$ mRNA levels between gastric type and pancreatobiliary type. When all intraductal papillary mucinous neoplasms were divided into intestinal type $(n=25)$ and non-intestinal type $(n=55), C L D N 4$ mRNA levels were significantly higher in intestinal type than in non-intestinal type $(P<0.0001$, Figure 4a). Furthermore, the comparison was confined to the high-grade neoplasms $(n=44)$, the expression levels of $C L D N 4$ mRNA were significantly higher in high-grade intestinal type $(n=25)$ than in high-grade non-intestinal type $(n=19)(P=0.0222$, Figure $4 b)$.

\section{Quantitative Analysis of CLDN4 mRNA Levels Between Invasive Carcinoma Derived from Intraductal Papillary Mucinous Neoplasm and Conventional Pancreatic Ductal Adenocarcinoma}

It is interesting to investigate the molecular difference between invasive carcinomas derived from intraductal papillary mucinous neoplasms and conventional pancreatic ductal adenocarcinomas because previous reports have shown a better prognosis in patients with invasive carcinoma derived from intraductal papillary mucinous neoplasm than in those with conventional pancreatic ductal adenocarcinoma. We, therefore, compared CLDN4 mRNA levels between 10 cases with invasive carcinoma derived from intraductal papillary mucinous neoplasm, including 2 cases of colloid type and 8 cases of tubular type, and 8 cases with conventional pancreatic ductal adenocarcinoma. The expression levels of CLDN4 mRNA in conventional pancreatic ductal adenocarcinomas were significantly higher than those in invasive carcinomas derived from intraductal papillary mucinous neoplasms (Figure 5, $P=0.0010$ ).

\section{Quantitative Analysis of CLDN4 mRNA Levels in Pancreatic Juice Samples}

To test whether quantitative measurement of CLDN4 expression in pancreatic juice could predict the malignant potential of intraductal papillary mucinous neoplasms, we examined CLDN4 mRNA in six pancreatic juice samples preoperatively obtained 

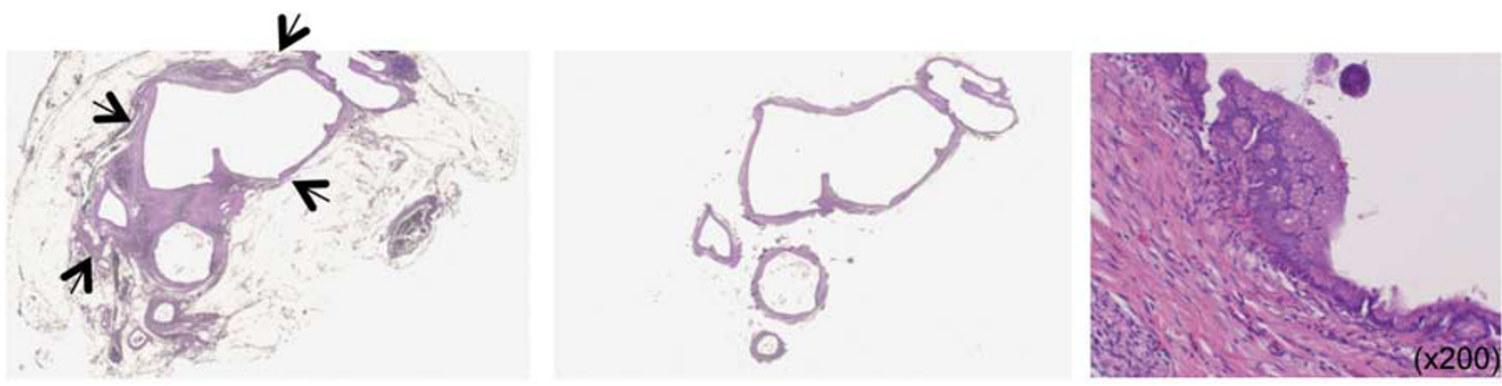

adenoma,

gastric type
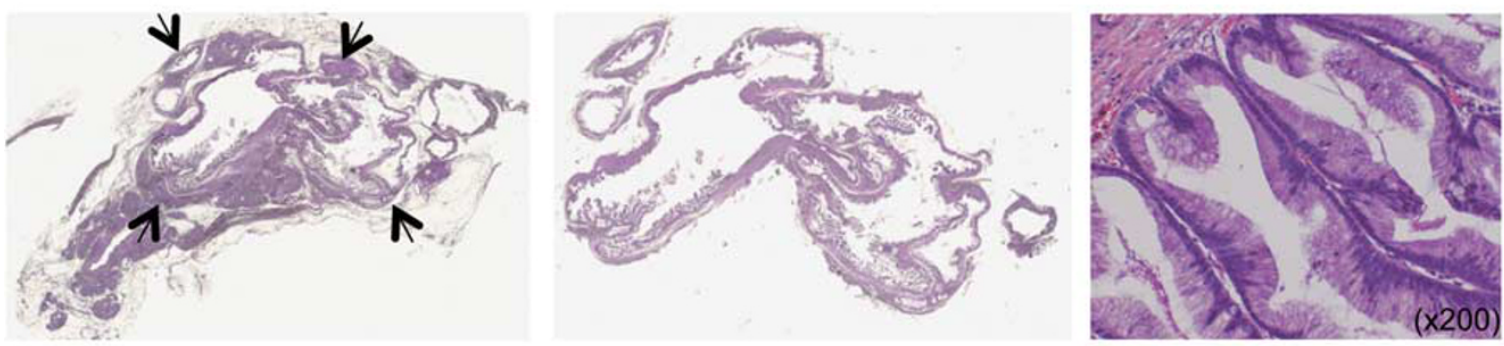

borderline, intestinal type
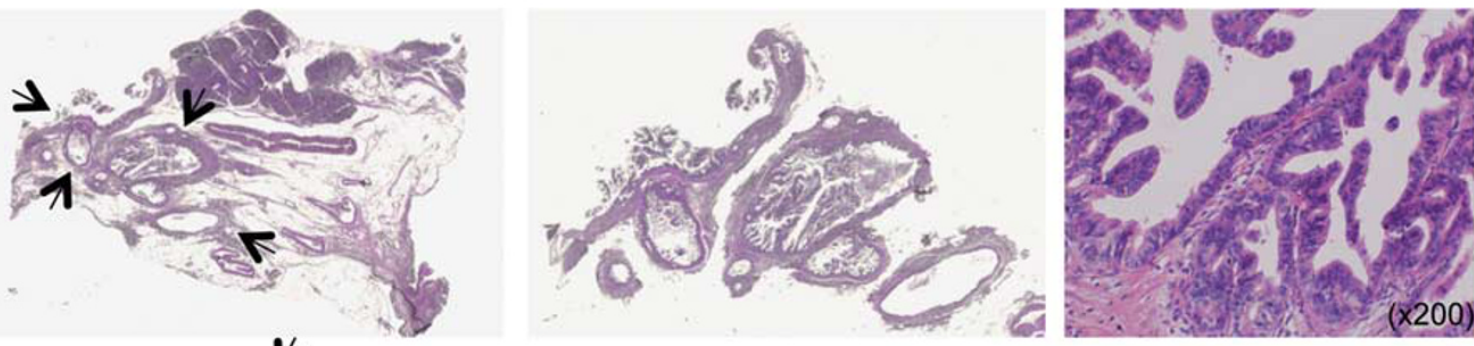

CIS,

pancreatobiliary type
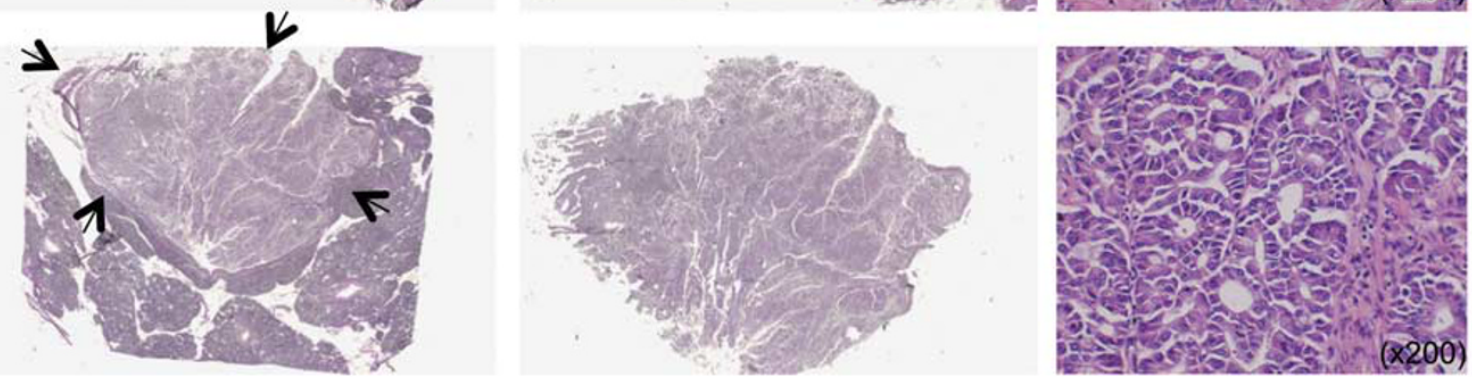

$\mathrm{CIS}$

oncocytic type

Figure 2 Representative pictures of macrodissection in formalin-fixed paraffin-embedded tissue samples (left and middle) and the photomicrographs of $\mathrm{H}$ and $\mathrm{E}$ staining (right). Original magnification: (left and middle) loupe, (right) $\times 200$. After identification of the distribution of neoplastic cells (indicated by black arrow), only the neoplastic component was selectively isolated as much as possible to obtain a high neoplastic cellularity using an electron microscope.

from patients with intraductal papillary mucinous adenoma $(n=3)$ and invasive carcinoma derived from intraductal papillary mucinous neoplasm $(n=3)$. The CLDN4 mRNA expression levels were significantly higher in pancreatic juice samples from patients with invasive carcinoma than in those from patients with adenoma (Figure 6, $P=0.0495$ ).

\section{Discussion}

This is the first report of quantitative analysis of CLDN4 mRNA expression in a large series of intraductal papillary mucinous neoplasms. We found (1) that CLDN4 expression was significantly higher in high-grade intraductal papillary mucinous neoplasms (borderline neoplasm \& carcinoma) than in low-grade intraductal papillary mucinous neoplasms (adenoma) $(P<0.0001),(2)$ that $C L D N 4$ mRNA levels were significantly higher in intestinaltype intraductal papillary mucinous neoplasms than in non-intestinal-type intraductal papillary mucinous neoplasms based on papillary subclassification $(P<0.0001)$, (3) that $C L D N 4$ mRNA expression was significantly higher in conventional pancreatic ductal adenocarcinomas than in invasive carcinomas derived from intraductal papillary mucinous neoplasms $(P=0.0010)$, and (4) that $C L D N 4$ mRNA expression levels were significantly higher in pancreatic juice samples from patients with invasive 
carcinoma derived from intraductal papillary mucinous neoplasm than in those from patients with intraductal papillary mucinous adenoma $(P=0.0495)$. These findings suggest that $C L D N 4$ may have an important role in the neoplastic progression of intraductal papillary mucinous neoplasms and represents a novel diagnostic and therapeutic target.
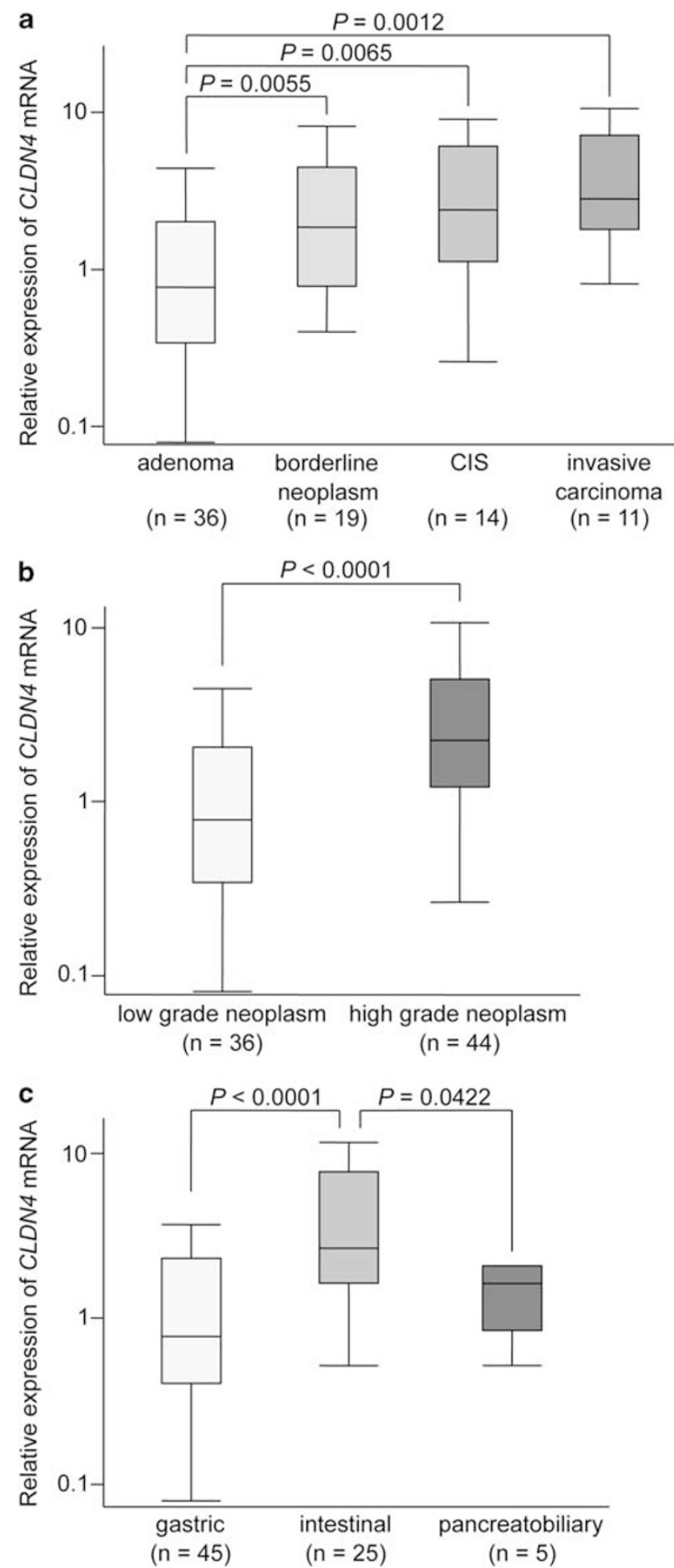

CLDN4 has been identified as overexpressed in pancreatic ductal adenocarcinomas by gene expression profiling. ${ }^{20,27}$ Immunohistochemical analysis revealed overexpression of CLDN4 protein in virtually all primary (99\%) and metastatic (100\%) pancreatic tissue samples and in 10 out of 11 samples of PanIN, a known precursor lesion to pancreatic ductal adenocarcinoma. ${ }^{21}$ Moreover, CLDN4 has been shown to be overexpressed in intraductal papillary mucinous neoplasms as well by gene expression profiling, ${ }^{14,28}$ although the expression analysis was limited within a small number of patients. In the present study, we demonstrated an increase in the CLDN4 mRNA expression along the progression of intraductal papillary mucinous neoplasms. Our results are consistent with a previous immunohistochemical analysis showing a positive correlation between CLDN4 protein expression and histological tumor grades in intraductal papillary mucinous neoplasms. ${ }^{29}$ The mechanism underlying the overexpression of CLDN4 in intraductal papillary mucinous neoplasms remains unclear, but a previous study has shown an association between CLDN4 overexpression and gene hypomethylation in pancreatic cancer cells. ${ }^{26}$

In the present study, we analyzed the relationship between CLDN4 expression and papillary subtypes of intraductal papillary mucinous neoplasms. Intraductal papillary mucinous neoplasms with different papillary subtypes have distinct histopathological features, clinical behaviors, and mucin profiles, suggesting that they may follow different

Figure 3 (a) $C L D N 4$ expression in intraductal papillary mucinous neoplasms according to the histological grade, which consist of adenoma $(n=36)$, borderline neoplasm $(n=19)$, CIS $(n=14)$, and invasive carcinoma $(n=11)$. The expression levels of CLDN4 mRNA in intraductal papillary mucinous borderline neoplasm (median, 1.85), CIS (median, 2.39), and invasive carcinoma derived from intraductal papillary mucinous neoplasm (median, 2.80) are significantly higher than those in intraductal papillary mucinous adenoma (median, 0.77) (adenoma versus borderline neoplasm; $P=0.0055$, adenoma versus CIS; $P=0.0065$, adenoma versus invasive carcinoma; $P=0.0012$, Wilcoxon rank-sum test). (b) Expression levels of CLDN4 mRNA between high-grade intraductal papillary mucinous neoplasms $(n=44)$ and low-grade intraductal papillary mucinous neoplasms $(n=36)$. High-grade intraductal papillary mucinous neoplasms mean borderline neoplasm, CIS and invasive carcinoma, while low-grade intraductal papillary mucinous neoplasms mean adenoma. There is a significant difference of CLDN4 mRNA levels between high-grade intraductal papillary mucinous neoplasms and low-grade intraductal papillary mucinous neoplasms $(P<0.0001$, Wilcoxon ranksum test). (c) CLDN4 expression in intraductal papillary mucinous neoplasms according to the papillary subtype, which consist of gastric type $(n=45)$, intestinal type $(n=25)$, pancreatobiliary type $(n=5)$. Intestinal type (median, 2.72) shows significantly higher level of CLDN4 mRNA than gastric type (median, 0.81) and pancreatobiliary type (median, 1.67) (gastric type versus intestinal type; $P<0.0001$, intestinal type versus pancreatobiliary type; $P=0.0422$, Wilcoxon rank-sum test). However, there is no difference of CLDN4 mRNA levels between gastric type and pancreatobiliary type. 

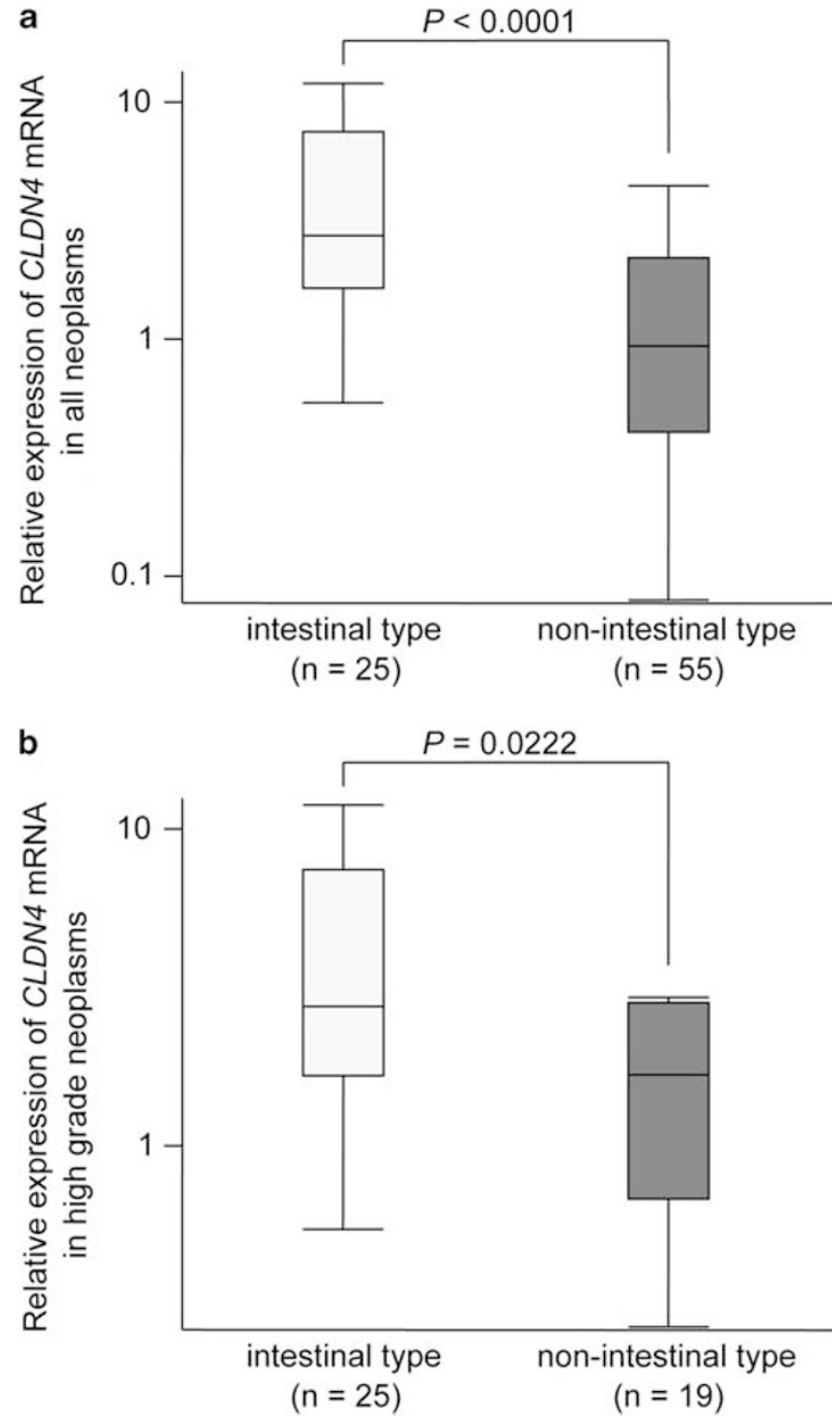

Figure 4 (a) Expression levels of CLDN4 mRNA between intestinal-type intraductal papillary mucinous neoplasms $(n=25)$ and non-intestinal-type intraductal papillary mucinous neoplasms $(n=55)$. Non-intestinal-type intraductal papillary mucinous neoplasms mean the gastric type, pancreatobiliary type, oncocytic type, and unclassified type. In all samples, there is a significant difference of CLDN4 mRNA levels between intestinal type and non-intestinal type $(P<0.0001$, Wilcoxon rank-sum test). (b) Expression levels of CLDN4 mRNA between high-grade-intestinal-type intraductal papillary mucinous neoplasms $(n=25)$ and high-grade-non-intestinal-type intraductal papillary mucinous neoplasms $(n=19)$. In high-grade intraductal papillary mucinous neoplasms, there is also a significant difference of CLDN4 mRNA levels between intestinal type and non-intestinal type $(P=0.0222$, Wilcoxon rank-sum test).

biological pathways. ${ }^{30}$ Recently, Sadakari and coworkers $^{3}$ have shown that invasive carcinoma derived from the non-intestinal-type intraductal papillary mucinous neoplasm has a poorer prognosis than that derived from the intestinal type. Previous studies have identified markers specific for intestinal-type intraductal papillary mucinous neoplasm, including MUC2, CDX2, and REG4. ${ }^{31}$ In the present study, we have identified CLDN4 as

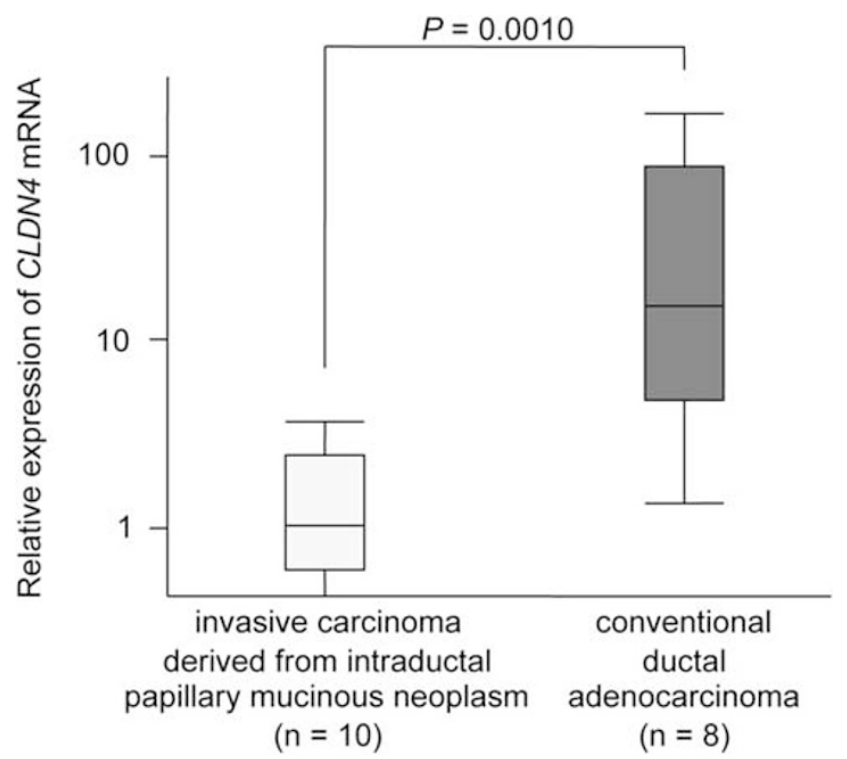

Figure $5 C L D N 4$ expression between invasive carcinoma derived from intraductal papillary mucinous neoplasm $(n=10)$ and conventional pancreatic ductal adenocarcinoma $(n=8)$. The expression levels of CLDN4 mRNA in conventional pancreatic ductal adenocarcinomas are significantly higher than those in invasive carcinomas derived from intraductal papillary mucinous neoplasm $(P=0.0010$, Wilcoxon rank-sum test).

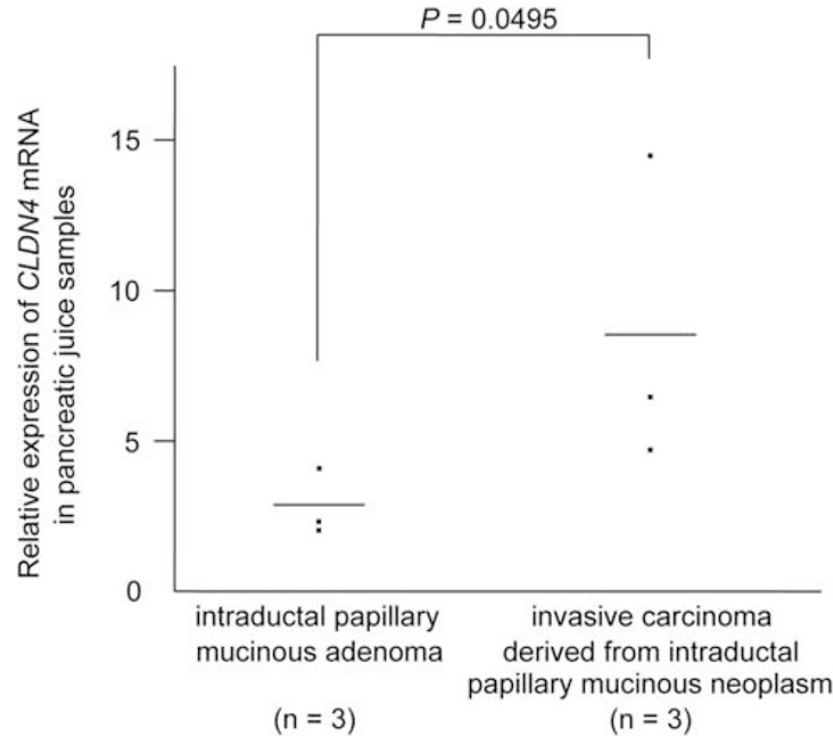

Figure $6 C L D N 4$ expression in pancreatic juice samples between intraductal papillary mucinous adenoma $(n=3)$ and invasive carcinoma derived from intraductal papillary mucinous neoplasm $(n=3)$. The $C L D N 4$ mRNA expression levels are significantly higher in pancreatic juice samples from patients with invasive carcinoma derived from intraductal papillary mucinous neoplasm than those from patients with intraductal papillary mucinous adenoma ( $P=0.0495$, Wilcoxon rank-sum test).

a novel marker for intestinal-type intraductal papillary mucinous neoplasm.

Frequent overexpression of CLDN4 in cancer has led to an idea that this molecule could be a target for cancer therapy. In fact, $C$. perfringens enterotoxin, 
for which CLDN4 is the receptor, has been shown to result in an acute dose-dependent cytotoxic effect in pancreatic cancer xenografts. ${ }^{18}$ More recently, a monoclonal antibody against $C L D N 4^{32}$ and C-terminal fragment of $C$. perfringens enterotoxin ${ }^{33}$ has been demonstrated to have antitumor activity in pancreatic cancer. These findings and our present results suggest that $C L D N 4$ represents a promising therapeutic target for pancreatic ductal adenocarcinomas and intraductal papillary mucinous neoplasms.

Our preliminary data suggests that quantitative analysis of CLDN4 mRNA in the pancreatic juice could be a marker to predict the presence of an associated invasive carcinoma in intraductal papillary mucinous neoplasms. Further investigation in a larger number of pancreatic juice samples is needed to determine the diagnostic role of CLDN4 in patients with intraductal papillary mucinous neoplasms.

In conclusion, our present findings suggest that CLDN4 expression is associated with neoplastic progression of intraductal papillary mucinous neoplasms and, especially, with a distinct pathway to intestinal differentiation.

\section{Acknowledgements}

We thank Miyuki Ohmori for expert technical assistance in preparing formalin-fixed paraffin-embedded sections for macrodissection, Midori Sato and Emiko Manabe for help in maintaining the cultures of cell lines. We appreciate the technical support from the Research Support Center, Graduate School of Medical Sciences, Kyushu University. This work was supported, in part, by a Grant-in-Aid from the Ministry of Education, Culture, Sports, Science and Technology of Japan.

\section{Disclosure/conflict of interest}

The authors declare no conflict of interest.

\section{References}

1 Longnecker DS, Alder G, Hruban RH, et al. Intraductal papillary-mucinous neoplasms of the pancreas. In: Hamilton SR, Aaltonen LA (eds). World Health Organization Classification of Tumours. Pathology and Genetics of Tumours of the Digestive System. IARC Press: Lyon, France, 2000, pp 237-240.

2 Tanaka M, Kobayashi K, Mizumoto K, et al. Clinical aspects of intraductal papillary mucinous neoplasm of the pancreas. J Gastroenterol 2005;40:669-675.

3 Sadakari Y, Ohuchida K, Nakata K, et al. Invasive carcinoma derived from the nonintestinal type intraductal papillary mucinous neoplasm of the pancreas has a poorer prognosis than that derived from the intestinal type. Surgery 2010;147:812-817.
4 Furukawa T, Klöppel G, Volkan Adsay $\mathrm{N}$, et al. Classification of types of intraductal papillarymucinous neoplasm of the pancreas: a consensus study. Virchows Arch 2005;447:794-799.

5 Andrejevic-Blant S, Kosmahl M, Sipos B, et al. Pancreatic intraductal papillary-mucinous neoplasms: a new and evolving entity. Virchows Arch 2007;451:863-869.

6 Tanaka $\mathrm{M}$, Chari S, Adsay $\mathrm{V}$, et al. International consensus guidelines for management of intraductal papillary mucinous neoplasms and mucinous cystic neoplasms of the pancreas. Pancreatology 2006;6:17-32.

7 Sessa F, Solcia E, Capella C, et al. Intraductal papillarymucinous tumours represent a distinct group of pancreatic neoplasms: an investigation of tumour cell differentiation and K-ras, p53 and c-erbB-2 abnormalities in 26 patients. Virchows Arch 1994; 425:357-367.

8 Laghi L, Orbetegli O, Bianchi P, et al. Common occurrence of multiple K-RAS mutations in pancreatic cancers with associated precursor lesions and in biliary cancers. Oncogene 2002;21:4301-4306.

9 Iacobuzio-Donahue CA, Klimstra DS, Adsay NV, et al. Dpc-4 protein is expressed in virtually all human intraductal papillary mucinous neoplasms of the pancreas: comparison with conventional ductal adenocarcinomas. Am J Pathol 2000;157:755-761.

10 Biankin AV, Biankin SA, Kench JG, et al. Aberrant p16(INK4A) and DPC4/Smad4 expression in intraductal papillary mucinous tumours of the pancreas is associated with invasive ductal adenocarcinoma. Gut 2002;50:861-868.

11 Sahin F, Maitra A, Argani P, et al. Loss of Stk11/Lkb1 expression in pancreatic and biliary neoplasms. Mod Pathol 2003;16:686-691.

12 Sato N, Ueki T, Fukushima N, et al. Aberrant methylation of $\mathrm{CpG}$ islands in intraductal papillary mucinous neoplasms of the pancreas. Gastroenterology 2002;123:365-372.

13 House MG, Guo M, Iacobuzio-Donahue C, et al. Molecular progression of promoter methylation in intraductal papillary mucinous neoplasms (IPMN) of the pancreas. Carcinogenesis 2003;24:193-198.

14 Sato N, Fukushima N, Maitra A, et al. Gene expression profiling identifies genes associated with invasive intraductal papillary mucinous neoplasms of the pancreas. Am J Pathol 2004;164:903-914.

15 Hough CD, Sherman-Baust CA, Pizer ES, et al. Largescale serial analysis of gene expression reveals genes differentially expressed in ovarian cancer. Cancer Res 2000;60:6281-6287.

16 Kominsky SL, Vali M, Korz D, et al. Clostridium perfringens enterotoxin elicits rapid and specific cytolysis of breast carcinoma cells mediated through tight junction proteins claudin 3 and 4. Am J Pathol 2004;164:1627-1633.

17 Long H, Crean CD, Lee WH, et al. Expression of Clostridium perfringens enterotoxin receptors claudin-3 and claudin-4 in prostate cancer epithelium. Cancer Res 2001;61:7878-7881.

18 Michl P, Buchholz M, Rolke M, et al. Claudin-4: a new target for pancreatic cancer treatment using Clostridium perfringens enterotoxin. Gastroenterology 2001;121:678-684.

19 Ryu B, Jones J, Blades NJ, et al. Relationships and differentially expressed genes among pancreatic cancers examined by large-scale serial analysis of gene expression. Cancer Res 2002;62:819-826. 
20 Iacobuzio-Donahue CA, Maitra A, Olsen M, et al. Exploration of global gene expression patterns in pancreatic adenocarcinoma using cDNA microarrays. Am J Pathol 2003;162:1151-1162.

21 Nichols LS, Ashfaq R, Iacobuzio-Donahue CA. Claudin 4 protein expression in primary and metastatic pancreatic cancer: support for use as a therapeutic target. Am J Clin Pathol 2004;121:226-230.

22 Hewitt KJ, Agarwal R, Morin PJ. The claudin gene family: expression in normal and neoplastic tissues. BMC Cancer 2006;6:186.

23 Morita K, Furuse M, Fujimoto K, et al. Claudin multigene family encoding four-transmembrane domain protein components of tight junction strands. Proc Natl Acad Sci USA 1999;96:511-516.

24 Sato N, Mizumoto K, Beppu K, et al. Establishment of a new human pancreatic cancer cell line, NOR-P1, with high angiogenic activity and metastatic potential. Cancer Lett 2000;155:153-161.

25 Ohuchida K, Mizumoto K, Murakami M, et al. Radiation to stromal fibroblasts increases invasiveness of pancreatic cancer cells through tumor-stromal interactions. Cancer Res 2004;64:3215-3222.

26 Sato N, Maitra A, Fukushima N, et al. Frequent hypomethylation of multiple genes overexpressed in pancreatic ductal adenocarcinoma. Cancer Res 2003;63:4158-4166.

27 Ryu B, Jones J, Hollingsworth MA, et al. Invasionspecific genes in malignancy: serial analysis of gene expression comparisons of primary and passaged cancers. Cancer Res 2001;61:1833-1838.

28 Terris B, Blaveri E, Crnogorac-Jurcevic $\mathrm{T}$, et al. Characterization of gene expression profiles in intraductal papillary-mucinous tumors of the pancreas. Am J Pathol 2002;160:1745-1754.

29 Tsukahara M, Nagai H, Kamiakito T, et al. Distinct expression patterns of claudin-1 and claudin-4 in intraductal papillary-mucinous tumors of the pancreas. Pathol Int 2005;55:63-69.

30 Adsay NV, Merati K, Basturk O, et al. Pathologically and biologically distinct types of epithelium in intraductal papillary mucinous neoplasms: delineation of an 'intestinal' pathway of carcinogenesis in the pancreas. Am J Surg Pathol 2004;28:839-848.

31 Nakata K, Nagai E, Ohuchida K, et al. REG4 is associated with carcinogenesis in the 'intestinal' pathway of intraductal papillary mucinous neoplasms. Mod Pathol 2009;22:460-468.

32 Suzuki M, Kato-Nakano M, Kawamoto S, et al. Therapeutic antitumor efficacy of monoclonal antibody against Claudin-4 for pancreatic and ovarian cancers. Cancer Sci 2009;100:1623-1630.

33 Kakutani H, Kondoh M, Saeki R, et al. Claudin-4targeting of diphtheria toxin fragment A using a C-terminal fragment of Clostridium perfringens enterotoxin. Eur J Pharm Biopharm 2010;75: 213-217.

Supplementary Information accompanies the paper on Modern Pathology website (http://www.nature.com/ modpathol) 